\section{Military Technical College Kobry El-Kobbah, Cairo, Egypt}

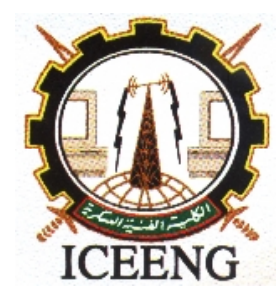

\section{$8^{\text {th }}$ International Conference on Electrical Engineering ICEENG 2012}

\title{
Tradeoffs for Selecting Orbital Parameters of an Earth Observation Satellite
}

\author{
E. Sh. Sanad ${ }^{1}$ \\ A. Y EL Raffie ${ }^{2}$ \\ Dr. F. Altohamy ${ }^{3}$ \\ Dr. M.A.Zayan ${ }^{4}$
}

\begin{abstract}
Selection of orbital parameters for earth observation satellites is the most reflective factor upon fulfillment of the space mission requirements. Practically, straight forward approach for selection of these parameters does not exist. Therefore, it is necessary to follow a complex process that requires tradeoffs among the different parameters and the corresponding orbit-related requirements. Multiple mission requirements often drive the orbit to different or even opposite trends. For Earth observation satellites, the most important orbit-related requirements are the Earth coverage, payload performance characteristics, ground communication, environment and survivability, orbit life time, availability of launch vehicle and legal and political constraints. In this work, analyses of how each orbital parameter would affect these requirements are introduced. Then the tradeoffs for compromising among contradictions are presented. To demonstrate with numerical example, a mathematical modeling for the correlations among altitude, revisit time, ground resolution and a single satellite slewing maneuver angle is developed. To implement for large scope of the mentioned parameters, a corresponding Matlab computer program is also developed. As an output, four parametric charts representing the correlations among altitudes, satellite slewing maneuver angle, ground resolution and the revisit time are obtained. The revisit time works as a parameter in these charts. Finally, a demonstrative tradeoff is introduced via those charts.
\end{abstract}

Keywords: [Orbital parameters, Earth observation, Earth coverage, satellite orbit design, satellite tracking, orbital altitude, and orbital revisit time].

\section{Introduction}

One of the most important bases of using space is detecting information about our planet earth and its atmosphere by remote sensing satellite systems. For this purpose, the specific operational orbit as one of the basic component of space mission plays a special role in satellite system design. It can be emphasized that the quality of a space mission is proportionally depending on its orbital selection[1]-[2]. Orbital selection (determination of its hexagonal parameters), in its turn is a faction of requirements specified for the mission. To design a mission orbit, it is required to determine the effect of the orbit parameters on key mission requirements. In this work first, we will introduce in brief the mission requirements and some related terminologies. Then we will analyze how each orbital parameter would affect these requirements. By extension the tradeoffs for compromising among contradictions are presented. After that we will introduce a mathematical model for the correlations among altitude, revisit time, ground resolution and satellite slewing maneuver angle. The computations are carried out by calculating minimal slewing maneuver angle of optical axis

1 M.SC., Student, M.T.C, Egyptian armed forces,ebrahemsanad@yahoo.com

2 Ph.D., Researcher in National Authority of Remote Sensing and Space Sciences, raffie_dyn@yahoo.com

3 Ph.D., M.T.C, Egyptian armed forces, ftohamy72@yahoo.com

4 Ph.D., Satellite Control Station Manager in Egyptian Satellites Company, Nilesat, m_zayan@yahoo.com 
for the satellite payload necessary to provide full coverage for specific latitude of earth surface with strips of possible imaging within specified time interval during Earth remote sensing process.

This technology can be used in space-based observation system's design to select the SC orbital parameters (altitude and inclination), range of axis slewing maneuver angle to achieve specified values of time parameters and coefficients of system's efficiency.

\section{Measures of performance}

Satellite orbit is determined by mission requirements and may sometimes be limited by launch restrictions. The imaging mission design goal is to achieve the largest amount of imagery with the best quality and the best coverage of areas for a longest time during satellite lifetime. Compromises of these requirements determine the satellite orbit.

The mission requirements related to orbital determination of Satellite movement in remote sensing Satellite Systems is categorized as five divisions as follows[3]:

1. Swath width and locations resolution accuracy of the Satellite payload equipment's for different geographical region of earth surface should be similar.

2. The Satellite should have a continuance from receiving information possibilities as much as possible of the earth surfaces. (In the other hand as much as possible of the earth surfaces should be covered).

3. Illumination conditions of earth surfaces facing the Satellite should be minimal during the Satellite payload equipment operational time.

4. The revisit time should be minimal.

5. Repeated coverage of a specific region of the earth surface should have a deterministic period.

The imaging quality is composed of the resolution and swath width, as well as the parameters related to illumination conditions. The area covered is defined by either as a requirement for global worldwide coverage or a requirement for frequent revisit of limited specific regions.

The best coverage is achieved by maximizing the frequency of imaging revisit, imaging velocity and the maximum swath width.

The survey areas accessible to an imager mounted on a satellite, as it travels around the earth, depend on the instrument field of view and on orbital parameters as altitude and inclination which determine the satellite trace over the surface of the earth[4]-[5]. The orbit to be used in any particular remote sensing mission has always been determined through a tradeoff between coverage objectives and the capabilities of the sequential trace pattern development, taking also into account the desired ground resolution[6]-[7].

Coverage missions usually fall into two general categories:

i. Complete intermittent survey of a given area during a relatively short period.

ii. Continuous surveillance of a target area.

A single satellite is able to yield coverage of the area for a few numbers of consecutive revolutions, creating a significant gap in coverage between them. This gap, less than the orbital period, can be covered either in a low repetition cycle by a large swath of the instrument or with a scarce temporal resolution if a better detail of observation is required. Temporal resolution (repeatability) refers to the temporal frequency with which a specific area can be imaged, by a remote sensing satellite payload, usually expressed in days. It depends on satellite's orbit characteristics and swath width (or payload FOV), the larger the swath (i.e. wider FOV at given orbit altitude), the higher is the temporal resolution (i.e. lower number of days)[8].

Spatial resolution (ground resolution) is denoted as the 'footprint' of a single detector element (pixel) on the ground through the optical unit of the payload. The spatial resolution is a measure of the payload's ability to image closely spaced objects so that they may be distinguished and recognized as separate objects, a payload with $1 \mathrm{~m}$ spatial resolution can reproduce finer details of the scene image compared to a payload with a $10 \mathrm{~m}$ spatial 
resolution. Therefore, a payload with $1 \mathrm{~m}$ spatial resolution is better than a payload with a $10 \mathrm{~m}$ spatial resolution $[3,8]$.

The best solution is obtained when the gap (either temporal or spatial) is reduced by using a satellite constellation that can consist of satellite deployed on one or more orbital planes [9] but it is not our interest in this work. This solution, in fact, has the potential of significantly improving the frequency of observation or the possibility of reducing the minimum spacing between ground tracks at the same repetition cycle of the single satellite. In this paper, we will work upon a single satellite by considering an orbit analysis for observation of Egyptian territories.

\section{Analysis and tradeoffs}

To design mission orbit it is required to determine the effect of the orbit parameters on key mission requirement. Table (1) summarizes the mission requirements that ordinarily determine the orbit parameters. The table shows that altitude is the most important of the orbit design parameter[1].

\begin{tabular}{|c|c|}
\hline Mission Requirement & Orbit Parameter \\
\hline $\begin{array}{l}\text { Coverage } \\
\text { Continuity } \\
\text { Frequency } \\
\text { Duration } \\
\text { Swath width } \\
\text { Ground track. } \\
\text { Area coverage rate } \\
\text { Slewing maneuver angles } \\
\text { Earth location of interest }\end{array}$ & $\begin{array}{l}\text { Altitude, Inclination, Node (only relevant for } \\
\text { some orbits as sun synchronous orbit) and } \\
\text { Eccentricity }\end{array}$ \\
\hline $\begin{array}{l}\text { Sensitivity or performance } \\
\text { Exposure or dwell time } \\
\text { Resolution }\end{array}$ & Altitude \\
\hline $\begin{array}{l}\text { Environment and survivability } \\
\text { Radiation environment } \\
\text { Lighting conditions } \\
\text { Hostile action }\end{array}$ & Altitude and Inclination \\
\hline $\begin{array}{l}\text { Launch capability } \\
\text { Launch cost } \\
\text { On-orbit weight } \\
\text { Launch site limitations }\end{array}$ & Altitude and Inclination \\
\hline $\begin{array}{l}\text { Ground communications } \\
\text { Ground station location } \\
\text { Data timeliness }\end{array}$ & Altitude, Inclination and Eccentricity \\
\hline Orbit lifetime & Altitude, Eccentricity \\
\hline $\begin{array}{l}\text { Legal or political constraints } \\
\text { Launch safety restrictions } \\
\text { International allocation }\end{array}$ & Altitude and Inclination \\
\hline
\end{tabular}

This table shows that the orbital altitude, inclination and eccentricity are affecting the various mission parameters as follows: 
- In general, moving the satellite higher results in better coverage, better survivability and easier communications. On the other hand, launch ability, resolution and payload weight tend to drive the satellite lower.

- For eccentricity, highly elliptical orbits give us non-uniform coverage and variable range and speed. This is besides the specialized launching requirements.

- Concerning orbital inclination, low inclination orbits provide limited coverage conditions than those of higher inclination ones. Also, a specified inclination requires special launching vehicle

The first step in finding the appropriate orbit for an earth-referenced mission is to determine if a specialized orbit will fit the mission. We should examine each of these orbits individually to see if its characteristics will meet the mission requirements at a reasonable cost. Because they do constrain such orbit parameters as altitude and inclination, we must determine whether or not to use them. Also, those specialized orbits have better launching opportunities examining of their fitness to the mission should be done before doing the more detailed design trades.

For an earth referenced orbit, specialized orbits are: geosynchronous, sun synchronous, frozen orbit, critical inclined, low inclined and repeating ground track orbits[10]. Analyses of application of those types of orbits are presented in the following.

For our mission, a sun synchronous orbit, repeating ground track, critical inclined and low inclined orbit will be required.

Sun synchronous orbit has the advantage of imaging with fixed illumination conditions for certain latitude, but it is required a retrograde orbit with high inclination angles, this reduces the density of the imagining area compared to lower inclination orbits.

For low inclined orbit, we have intensive imaging for the confined area, on the other hand loosing of sun synchronous condition can obscure imaging of certain area for long period can reach more than 15 days.

For critical inclined orbit we may have a convenient altitude for imagining certain location but this will cost more for injection a satellite in this orbit. As launch vehicle required getting elliptical orbit with certain perigee should be more powerful than having this perigee as a radius of circular orbit.

Assuming a circular orbit, this will limit our task to select either the altitude or the inclination of the orbit or both according to the selected type of orbit. This process establishes a range of altitudes and inclinations, from which we can select one or more alternatives.

- Ordinarily, low altitudes achieve better instrument performance because they are closer to the earth's surface. They also require less energy to reach orbit. 
- On the other hand higher orbits have longer lifetimes and provide better earth coverage.

- Aiming to have a satellite without propulsion system makes the orbital decay to be one of the most important drivers in selecting orbital altitude.

- Often a key factor in altitude selection is the satellite's radiation environment. The radiation environment undergoes a substantial change at approximately $1,000 \mathrm{~km}$. below this altitude the atmosphere will quickly clear out charged particles, so the radiation density is low. Above this altitude are the Van Allen Belts, whose high level of trapped radiation can greatly reduce the lifetime of spacecraft components.

- Most mission orbits separate naturally into either low earth orbit (LEO), below 1000 to $500 \mathrm{~km}$, and geosynchronous orbits (GEO), which are well above the Van Allen belts.

- Assuming an eccentric orbit, we assess the potential advantages of using eccentric orbits. These orbits have a greater peak altitude for a given amount of energy, lower perigee than is possible with a circular orbit, and lower velocity at the apogee, which makes more time available there. Unfortunately eccentric orbits also give us non-uniform converge and variable range and speed.

- Also, eccentric orbit have an additional difficulty because the oblateness of the earth cause perturbations which make perigee rotate rabidly. This rotation leads to rapid change in the apogee's position relative to the earth surface. Thus, with most orbits, we cannot maintain apogee for long over a given latitude, the first order rotation of perigee is proportional to $\left(2-2.5 \sin ^{2} i\right)$ which equal zero at an inclination, $i=63.4 \mathrm{deg}$. At this critical inclination the perigee will not rotate, so we can maintain apogee and perigee over fixed latitudes. Because this orientation can provide coverage at high northerner latitudes, the Soviet Union has used such a Molniya orbit for communications for many years. Geosynchronous orbits do not provide good coverage in high latitude regions. Table. 2 summarize some of altitude trades.

Table 2.Altitude trades

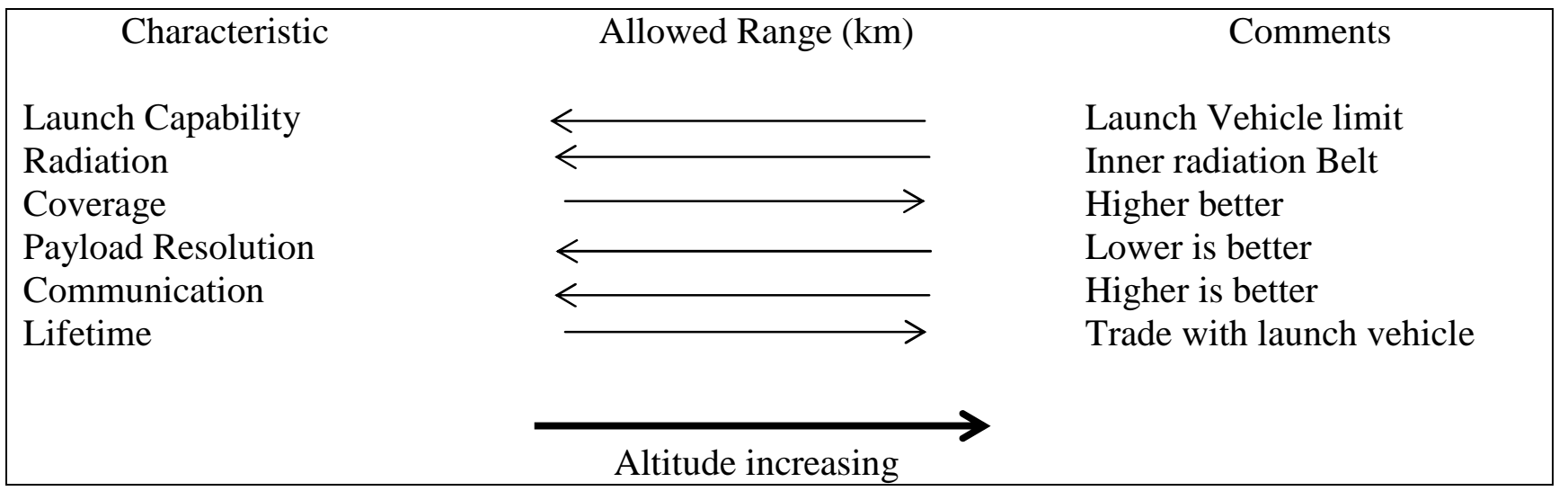




\section{Demonstrative example for orbital altitude selection}

In this section, we demonstrate the selection of orbital altitude for an earth observation satellite equipped with an optical payload. The main mission of the assumed satellite is to image the Egyptian territories as many times as possible during its lifetime of 3 years. Referring to the section of the specialized orbits, a sun-synchronous SSO orbit is providing the advantage of imaging of the Egyptian territories with closely similar illumination conditions. Selecting of this orbit allows achieving the mentioned advantage but not necessarily allows imaging as many times as possible to an object in this area. This is because; the width of the Egyptian territories is too large to be covered with any field of view of optical imager and the required high inclination angle of SSO. Hence, as many times as possible imaging of an object in this area will not be possible without slewing maneuver of the satellite. This imposes the requirements of the ground resolution of the received images to be ranging between the best cases for nadir imaging and worst one for the maximum slewing angle. Limiting our selection to SSO better surveillance of the Egyptian territories could be obtained through avoidance of repeated track orbit as it will be explained in the criteria for selection.

In Thus, the following demonstrative example we are going to select the orbit altitude to reach the following requirements:

- Certain ground resolution.

- Accepted worst ground resolution associated with maximum slewing maneuver angle.

- Minimum slewing angle.

- Minimum revisit time.

In our case an aerial revisit time is adopted. It is defined as the minimum number of days required to carry out the next image the same object in the Egyptian territories.

This section contains description of the method with the help of a flowchart for the Matlab program and illustrates the results in four parametric charts representing the relations among altitude, satellite slewing maneuver angle, ground resolution and revisit time.

\subsection{Method of calculation}

This program is developed based on the equation stated in reference [11].

To calculate minimal slewing maneuver angles necessary for full coverage with strips of possible imaging specified latitude, the procedure presented in the input the flowchart shown in figure (6) is applied as follows.

Then For every value of satellite altitude $h$ in altitude range $\left(h_{\min }\right.$ to $\left.h_{\max }\right)$ using step $(\Delta h)$ calculate the following steps[11]:

1. Earth radius corresponding to a specified latitude:

$$
\begin{aligned}
& R_{E}=R_{E e} \cdot\left(1-\frac{R_{E e}-R_{E p}}{R_{E e}} \cdot \sin ^{2}(\varphi)\right) \\
& \text { Where, } R_{E e} \quad \text { is the equatorial radius of Earth, } R_{E e}=6378.140 \mathrm{~km} . \\
& R_{E p} \quad \text { is the polar radius of Earth, } R_{E p}=6356.770 \mathrm{~km} .
\end{aligned}
$$

2. Angular value for calculation step using geographical longitude corresponding to a specified geographical latitude:

$$
\Delta \lambda=\frac{\Delta L}{R_{E} \cdot \cos (\varphi)}
$$

3. Radius of satellite orbit:

$$
\mathrm{r}=\mathrm{R}_{\mathrm{E}}+\mathrm{h}
$$

4. The sun synchronous orbit's inclination of the SC is calculated:

$$
i=\arccos \left(-\frac{2 \pi \cdot \sqrt{\mu} \cdot r^{7 / 2}}{\varepsilon \cdot T_{S}}\right)
$$


Where, $(\mu)$ Earth gravitational parameter, $\mu=398600.5 \mathrm{~km}^{3} / \mathrm{s}^{2}$.

( $\varepsilon$ ) parameter, characterizing polar flattening of Earth, $\varepsilon=2.6333 \times 1010 \mathrm{~km} / \mathrm{s}^{2}$

$\left(\mathrm{T}_{\mathrm{s}}\right)$ Period of Earth's rotation around Sun, $\mathrm{T}_{\mathrm{s}}=365.2422 \times 86400 \mathrm{sec}$.

5. Draconic period of the satellite revolution:

$T_{\Omega}=\frac{2 \pi}{\sqrt{\mu}} \cdot r^{3 / 2} \cdot\left\{1-\frac{\varepsilon}{\mu \cdot r^{2}}\left[3-\frac{5}{2} \cdot \sin ^{2}(i)\right]\right\}$

6. Period of Earth rotation in relation to the precession plane of satellite orbit:

$$
\mathrm{T}_{\mathrm{E} . \mathrm{rel}}=\frac{2 \pi}{\frac{2 \pi}{\mathrm{T}_{\mathrm{E}}}+\frac{\varepsilon \cdot \cos (\mathrm{i})}{\sqrt{\mu} \cdot \mathrm{r}^{7 / 2}}}
$$

Where $\mathrm{T}_{\mathrm{E}} \quad$ is the sidereal period of Earth rotation, $\mathrm{T}_{\mathrm{E}}=86164 \mathrm{~s}$.

7. Track's shift along longitude within one orbital revolution of the SC (track's shift between successive orbits):

$\lambda_{\text {orbit }}=2 \pi \frac{T_{\Omega}}{T_{E . r e l}}$

8. Track's shift along longitude within (int $\left[\mathrm{T}_{\mathrm{Erel}} / \mathrm{T}_{\Omega}\right]+1$ ) orbital revolutions of the $\mathrm{SC}$ (track's daily shift in given direction), where int $[. .$.$] is the integer part of a number:$

$\lambda_{\text {DAY }}=\lambda_{\text {orbit }}-\left(2 \pi-\lambda_{\text {orbit }} \cdot \operatorname{int}\left[\frac{2 \pi}{\lambda_{\text {orbit }}}\right]\right)$

9. Assume that maximal allowable slewing maneuver angle of imager optical axis is:

$$
\gamma_{\max }=\arcsin \left(\frac{\mathrm{R}_{\mathrm{E}}}{\mathrm{r}}\right)
$$

10. Central earth angle, corresponding to detector optical axis slewing maneuver angle:

$$
v=\arcsin \left(\frac{r}{R_{E}} \sin \left(\gamma_{\text {min }}\right)\right)-\gamma_{\text {min }}
$$

11. Angular value for that part of a latitude which is covered with one strip of possible imaging:

$$
\lambda_{O B S}=\arcsin \left(\frac{\sin (|\varphi|) \cdot \cos (i)+\sin (v)}{\cos (|\varphi|) \cdot \sin (i)}\right)-\arcsin \left(\frac{\sin (|\varphi|) \cdot \cos (i)-\sin (v)}{\cos (|\varphi|) \cdot \sin (i)}\right)
$$

For the selected special case of SSO, the coverage of single path of the satellite will be considered for the daylight path only (on the ascending orbit or on the descending orbit).

This single path coverage represented by $\left(\lambda_{O B S}\right)$ is illustrated with the associated angles in figure (1).

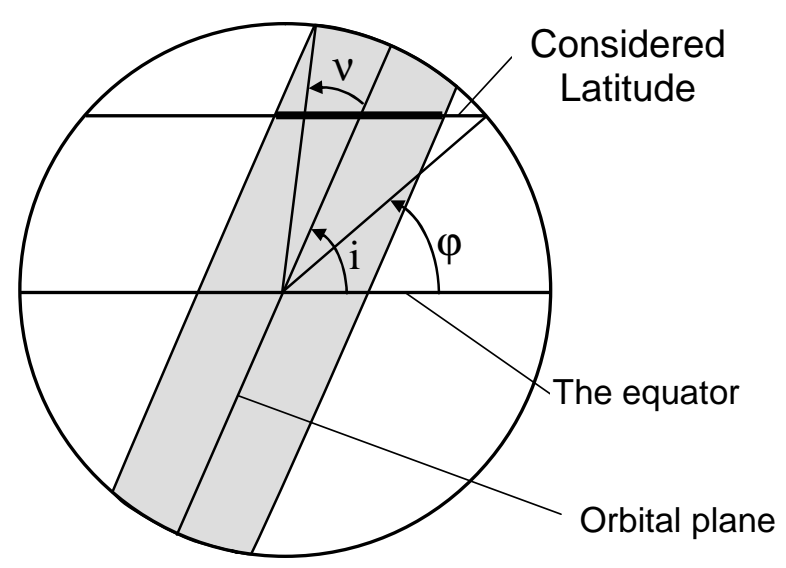

Fig. 1. The shaded area representing $\left(\lambda_{O B S}\right)$ with its associated angles 
By using the above equations with the help of the following flowchart shown in figure (6) we can solve this problem, and then we can get the following results.

\subsection{Results and illustrated charts}

The following Figures show calculation results of following dependence from satellite orbit altitude range between $(550 \mathrm{~km}-750 \mathrm{~km})$, when imaging specific latitude $\left(22^{\circ} \mathrm{N}\right)$ and revisit time for (2, 5, 20, 30 and 50 days) are described as follows:

Figure (2) is demonstrated for altitude range and slewing maneuver angle of the satellite payload for the cases of revisit time $(2,5,20,30$ and 50 days). Varying of the slewing maneuver angle for each altitude to reach the full coverage of the altitude circle and finding out the corresponding revisit time. In this figure the relation between maximum slewing maneuver angle and the altitude with the revisit time as a parameter is illustrated.

In figure (3) the slewing maneuver angle is fixed and the relation between altitude and the revisit time is evaluated. In this figure the relation between altitude and the revisit time with slewing maneuver angle as a parameter is illustrated.

Besides the previously mentioned two graphs, the following two graphs are also presented:

The relation between the altitude and the worst ground resolution with the revisit time as a parameter is illustrated in figure (4); the worst ground resolution is obtained at the strip edges due to the required slewing maneuver angles.

In figure (5) the relation between the altitude and the worst and best ground resolution for the selected revisit time (50 days) is illustrated.

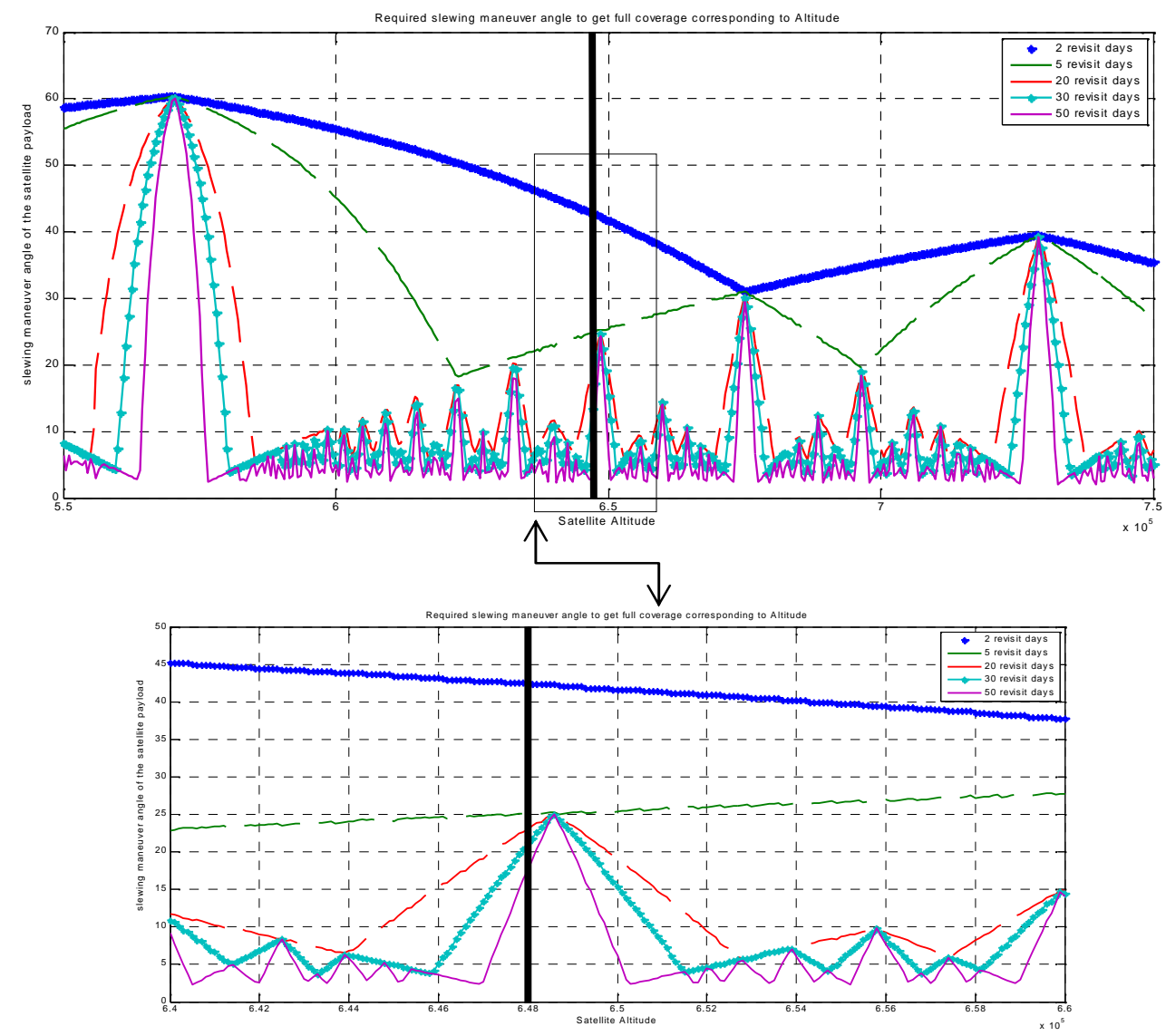

Fig. 2 
Proceedings of the $\boldsymbol{8}^{\text {th }} \mathbf{I C E E N G}$ Conference, 29-31 May, 2012

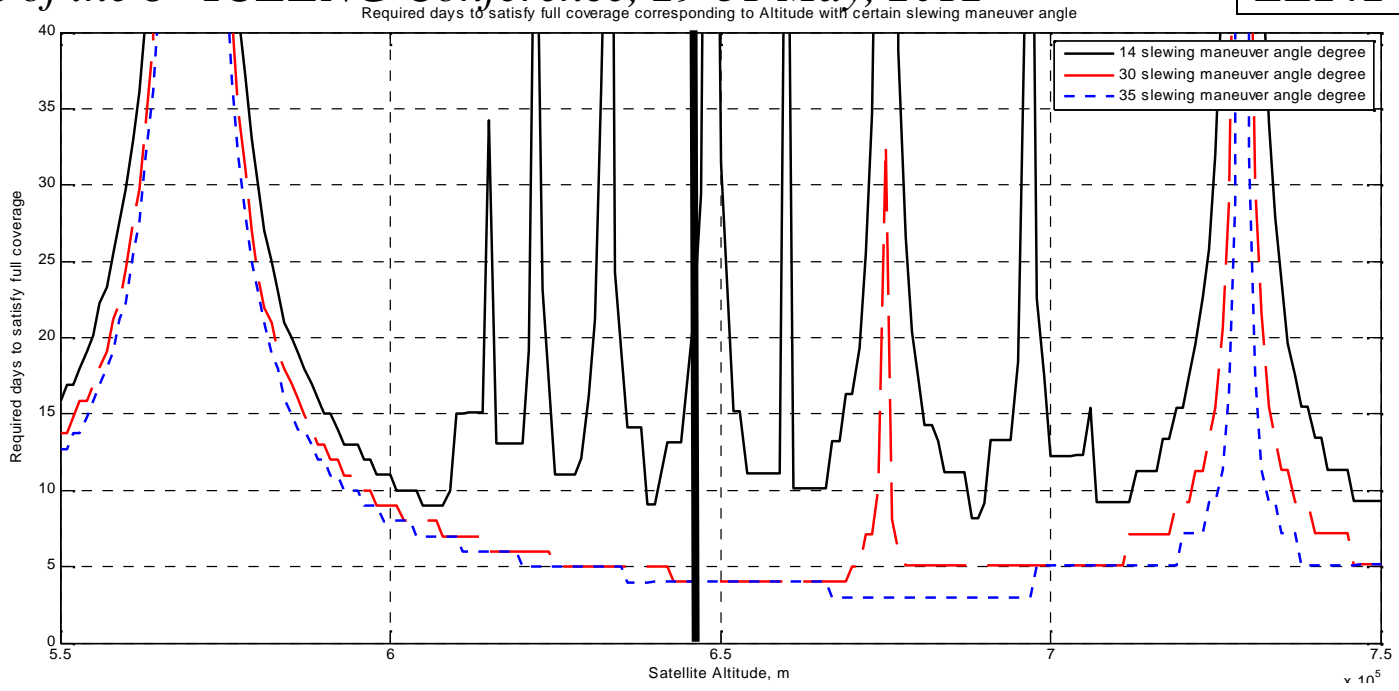

Fig. 3

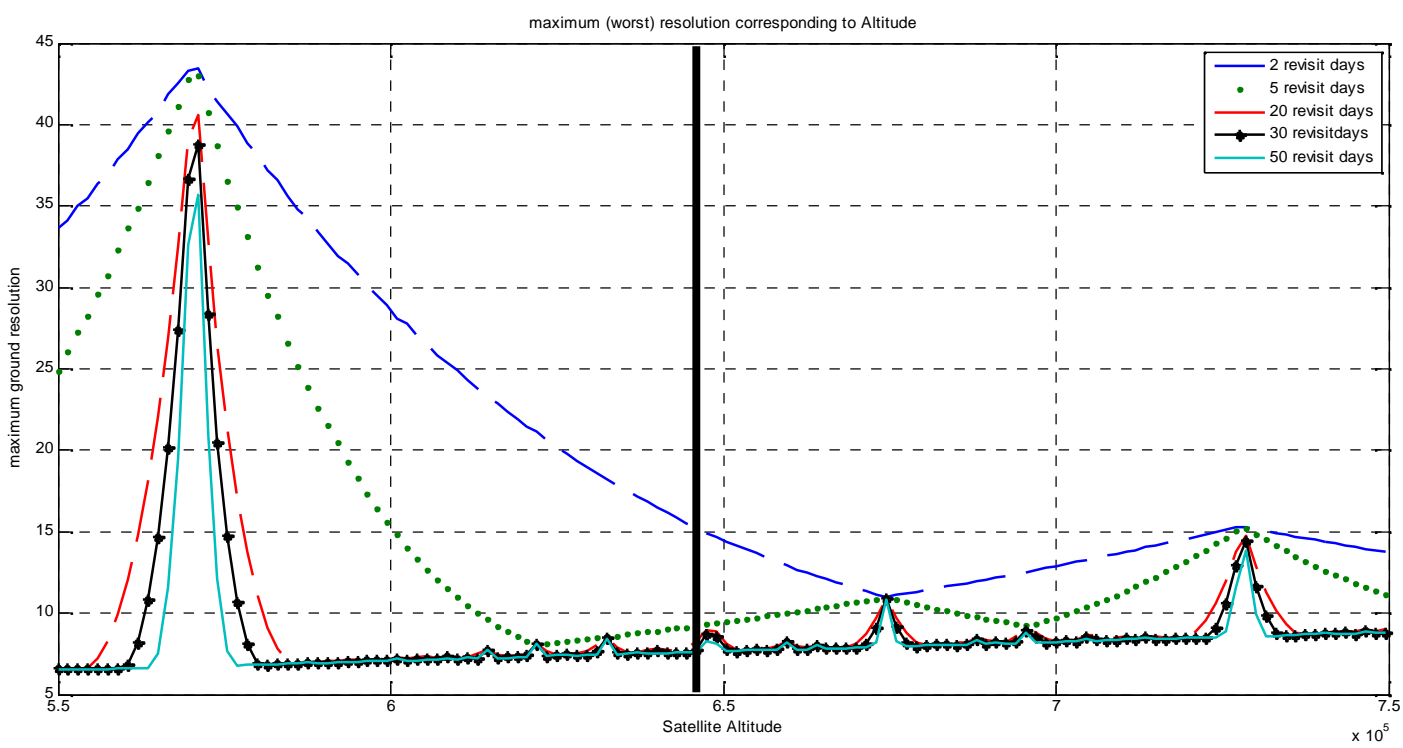

Fig. 4

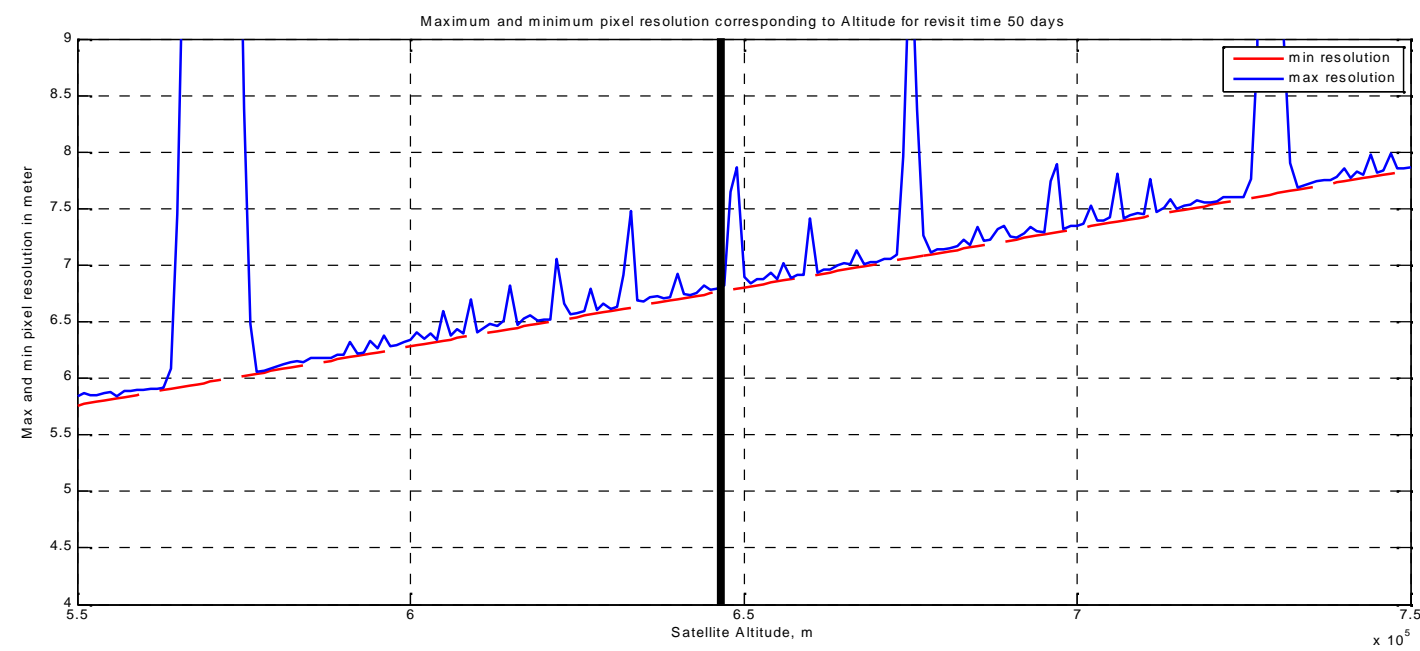

Fig. 5 


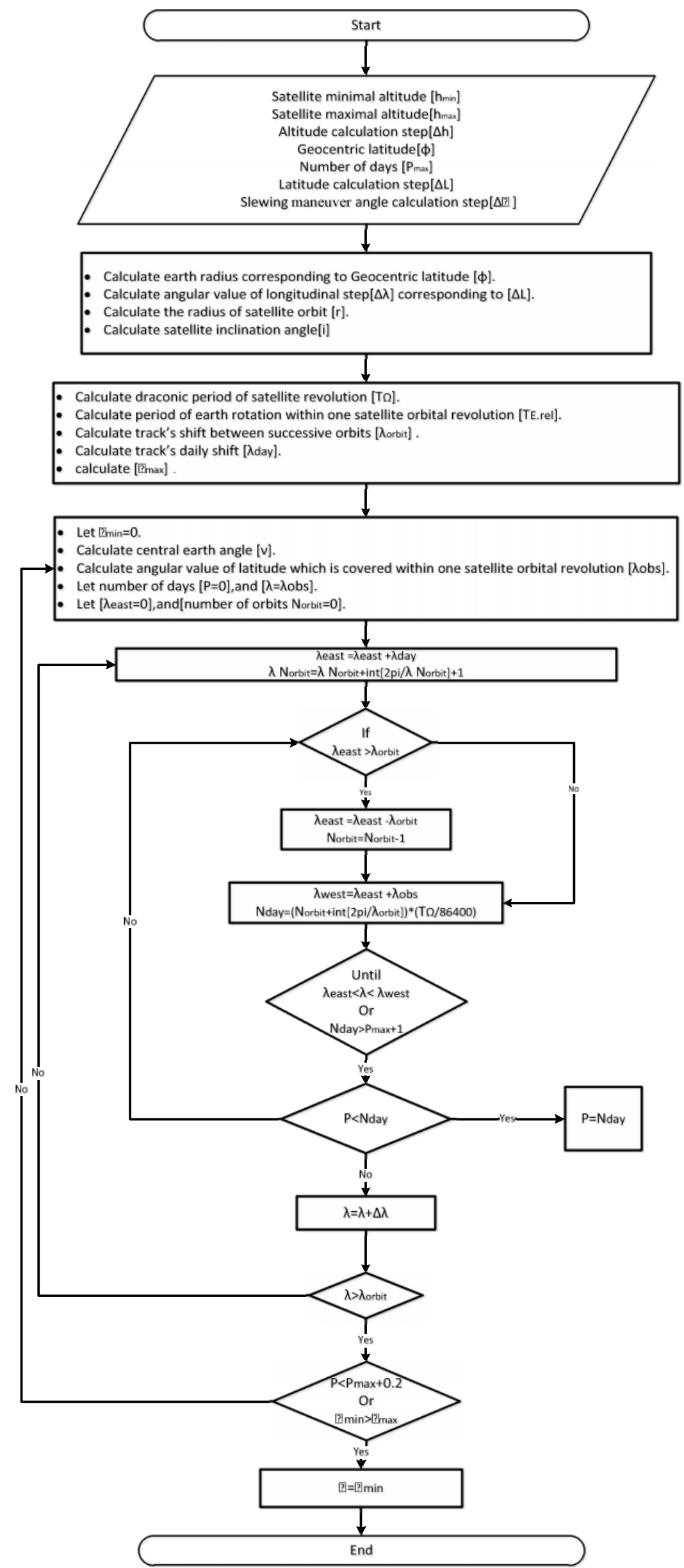

Fig. 6 . Program flowchart 


\subsection{Orbital altitude selection}

The four previously mentioned graphs are arranged above each other within the same order of numbering, where the altitude is the horizontal axis. To select the orbital altitude, the following requirements are assumed:

1. The best ground resolution is better than 8 meters.

2. The accepted worst ground resolution is 10 meters.

3. The revisit time is $\mathbf{5 0}$ days.

4. The slewing maneuver angle of the satellite camera is lower than 8 degrees.

5. The error due to L.V. is $\pm 6 \mathrm{~km}$.

6. The orbital life time is 3 years.

7. The altitude decay will be $30 \mathrm{~km}$.

From analysis of the upper graphs we see that any repeated track orbit requires tilting of the satellite to get off nadir imaging to improve the coverage requirements. It is impossible to avoid repeated ground track orbits, especially those of long period. Hence we use this program to select the orbital altitude that avoids short time repeated ground track orbits.

In the above graphs the peaks for either slewing maneuver angles or ground resolutions are representing the repeated ground tracks orbital altitudes.

From figure (2) and (4) we can select the orbital altitude according to two main criteria:

- Complete coverage with minimum slewing maneuver angle.

- Having better resolution

We know that when this angle is increased, the swath width increases, but at the expense of degrading the ground resolution at the strip edges. When the satellite operating orbit altitude nominal value is selected, the accuracy of injection of the launch vehicle (LV) must be taken into consideration. Proposed to inject the satellite Dniepr-1 LV provides injection accuracy by altitude $\pm 6 \mathrm{~km}$. Also we should take into consideration the altitude decay along the orbital life time. By preliminary estimation orbit decay can make from 3 to $35 \mathrm{~km}$ according to satellite life time will be 5 years.

After analysis of the upper graphs we may choose the following altitudes; 570, 630, 654, 675, and $715 \mathrm{~km}$ that give the following data as shown in table (3).

Table 3 Results for the selected altitudes

\begin{tabular}{|c|c|c|c|}
\hline $\begin{array}{c}\text { Altitude } \\
(\mathrm{km})\end{array}$ & $\begin{array}{c}\text { Slewing maneuver } \\
\text { angle (degrees) }\end{array}$ & $\begin{array}{c}\text { Min. (Best) ground } \\
\text { resolution in meters }\end{array}$ & $\begin{array}{c}\text { Max. (Worst) ground } \\
\text { resolution in meters }\end{array}$ \\
\hline 570 & 59.6 & 5.96 & 40.97 \\
\hline 630 & 2.8 & 6.59 & 6.61 \\
\hline $\mathbf{6 5 4}$ & $\mathbf{6 . 1}$ & $\mathbf{6 . 8 4}$ & $\mathbf{6 . 9 3}$ \\
\hline 675 & 29.4 & 7.06 & 9.84 \\
\hline 715 & 2.6 & 7.48 & 7.49 \\
\hline
\end{tabular}

After analyzing the previous selected results in table (3) we can choose the orbital altitude of $654 \mathrm{~km}$. proposed to inject the satellite Dniepr-1 LV provides injection accuracy by altitude $\pm 6 \mathrm{~km}$. Then the selected orbital altitude will be $\mathbf{H}=\mathbf{6 4 8} \mathbf{~ k m}$. For the selected altitude value of major semi major axis will be $\mathrm{a}=7026.14 \mathrm{~km}, \mathrm{SSO}$ inclination (i) $\approx 97.98^{\circ}$ and satellite revolution draconic period $\mathrm{T}=5861.19 \mathrm{sec}$. the minimal ground resolution of this orbit is 6.78 meter. Also we must take into consideration the altitude decay which improves the ground resolution along the period of the satellite life time (3 years) till it will reach to 6.48 meters at altitude of $620 \mathrm{~km}$. 
The following graph represents the satellite ground track for revisit time (50 days) produced by verification of this orbit altitude using the STK program.

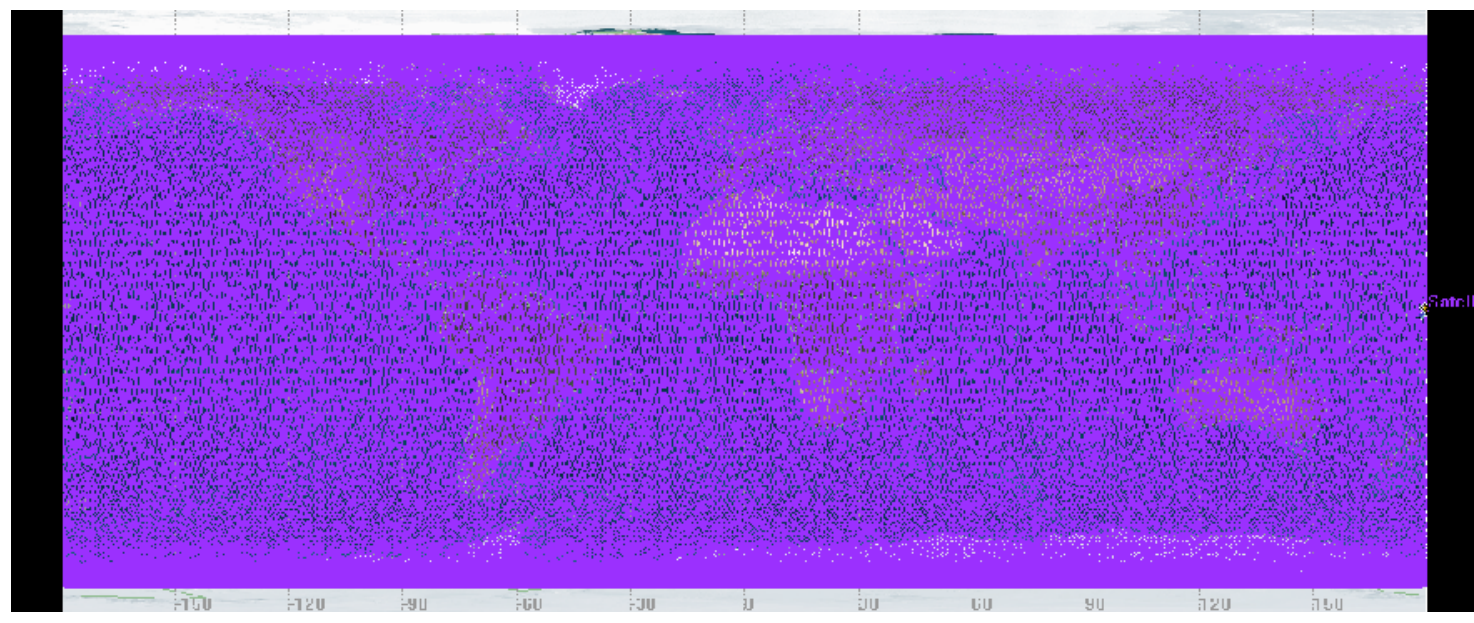

Fig. 7 Satellite ground tracks

\section{Conclusion}

In present article with categorizing the earth remote sensing satellite system requirements, an orbit design analysis oriented to obtain efficient revisit coverage and repeat cycles by instruments embarked on satellite which will be deployed on a Sun-synchronous orbit. Orbital parameters evolution is selected for 3 years.

\section{References}

[1] J. R. W. W.J. Larson, Space Mission Analysis and Design vol. 2th Edition, 1992.

[2] C. D. Brown, "Spacecraft Mission Design," AIAA Edition Series, 1992.

[3] M. M. Asghar Ebrahimi, S. Ali A. Moosavian, "ORBIT ANALYSIS OF A REMOTE SENSING SATELLITE FOR LOCAL OBSERVATION OF THE EARTH SURFACE."

[4] J. W. s. Marshall H. Kaplan, Modern Spacecraft Dynamics and Control, 1976.

[5] L. G. Ulivieri C., ands.M. Hejazi M. , "Small Satellite Constellations for Continuous Regional Surveillance," 12th Int. Symposium on Space Flight Dynamics, Germany, Jun 1997.

[6] B. M. V. Bobronnikov, "Mission Planning for Remote Sensing Satellite Constellation," 1998.

[7] H. R.G., "Long-Term Revisit Coverage Using Multi-Satellite Constellations," AIAA Paper, 1988.

[8] M. M., "Determination of Small Satellite Orbits for Earth Observation Missions," International Symposium of the International Academy of Astronautics (IAA), 2002.

[9] J. I. F. Grishin S.D., Lysovoi V.T., "Space Systems for Earth Remote Sensing," 1993.

[10] E. O. M., "REMOTE SENSING SATELLITES ORBITS DESIGN AND CONTROL."

[11] "Ukrainian document EGSYZHMAN13000E1 of the project EGYPTSAT-1." 